\title{
May two transcripts be enough to discriminate between Viral and Bacterial diarrhea?
}

Barral-Arca R ${ }^{1,2}$, Pardo-Seco J ${ }^{1,2}$, Martinón-Torres $\mathbf{F}^{2}$, Salas A 1 ,

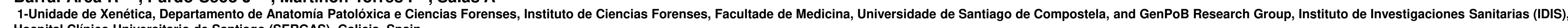

\section{Questions \\ Can the 2 transcripts (FAM89A and IFI44L) signature discovered by Herberg et al (2016) be used to distinguish viral from bacterial diarrhea? \\ Is the performance of the two transcript signature affected by the populational background of the patient?}

\section{Abstract}

2016 a biomarker signature consisting of 2-transcript host RNAs was designed for discerning bacterial from viral infections in febrile children (Herberg, 2016). We studied the performance of this 2 -transcript test in a real-
like discase scenario, that is the evaluation of its predictive capacity in a cohort of Mexican cohort children
(n=174) children with the same phenotype, acute diarrhea, caused by difiterent microorganisms. As ancestry is a known potential modifier of gene expression, we first examined the admixed background of the of European background (ranging from $0 \%$ to $57 \%$.). Our results confirm that the 2 -transcript test can distinguish
between viral and bacterial cause of infection (t-test; P-value $=6.94 \times 10^{-11} ; \mathrm{AUC}=80 \%$; sensitivity: $68 \%$ (95\% $\mathrm{C}$

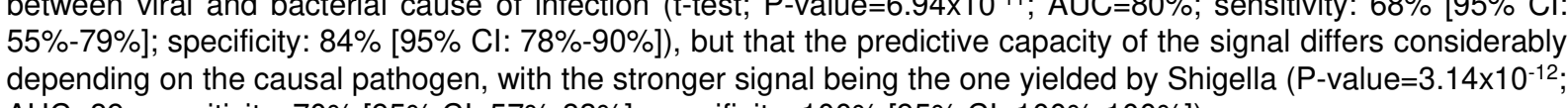
AUC=89, sentilily:

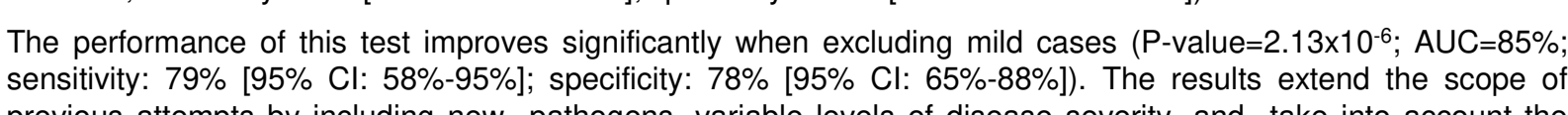
previous attemptst by including new pathogens, variable levels of disease severity, and take into account the
different ancestral background of patients. These results give confirmatory support to the clinical utility of this 2 -

\section{Material and Methods}

We retrieved from the GEO database (GSE69529) an RNA expression dataset obtained from
cohort $(n=174$ ) of Mexican children, having less than 10 years old, suffering from acute having cohort $(n=174)$ of Mexican children, having less than 10 years old, suffering from acuute having
diarrhea associated with a single viral or bacterial pathogen and without systemic complications Software employed

RNA-seq data analysis: STAR, FASTAQC,MultiQC, R (packages Deseq2, pROC, beeswarn)

Ancestry Analysis: Opossum, Platypus, PLINK, Admixture, R

Variant Calling and ancestry analysis from RNA-seq data
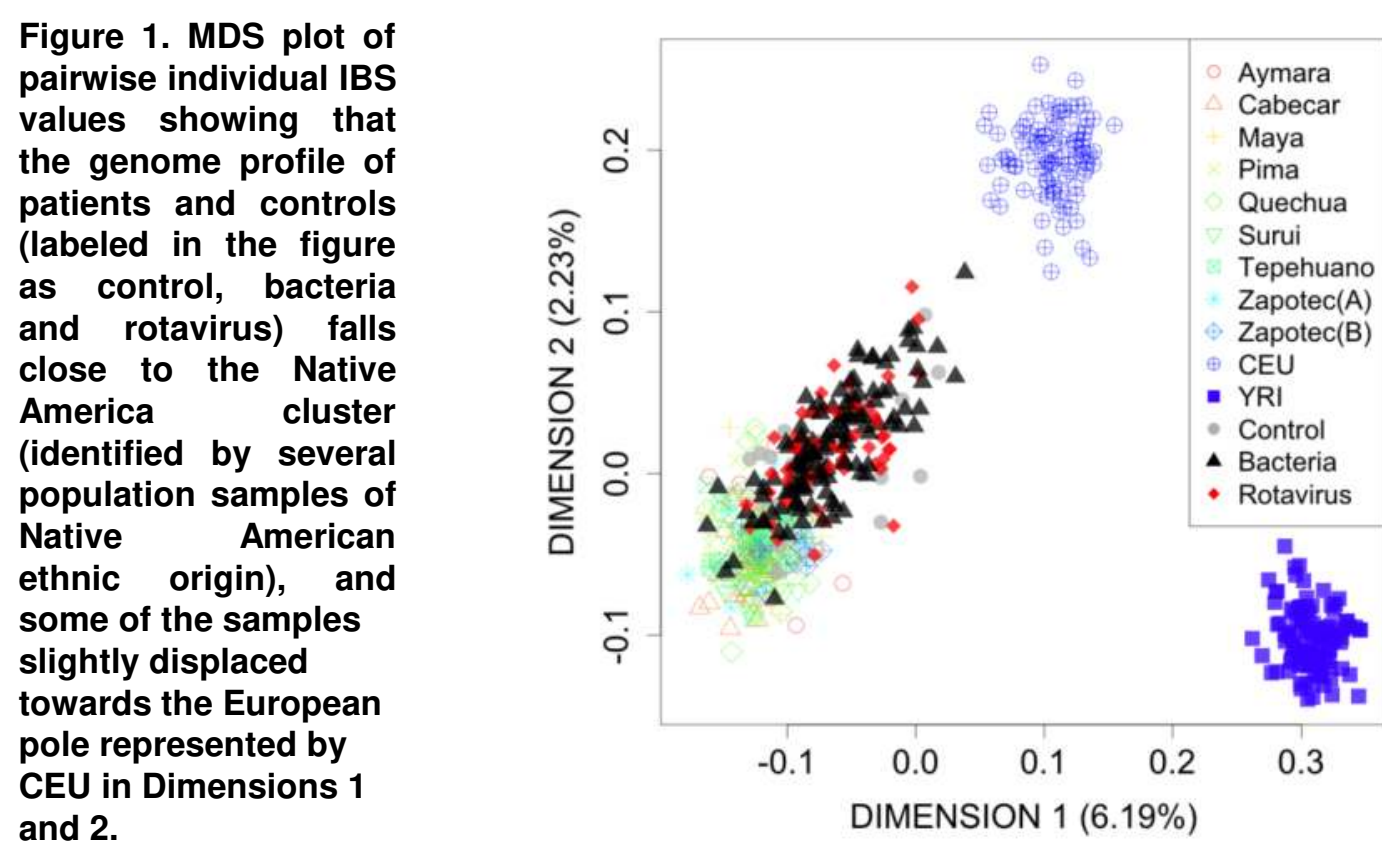

A

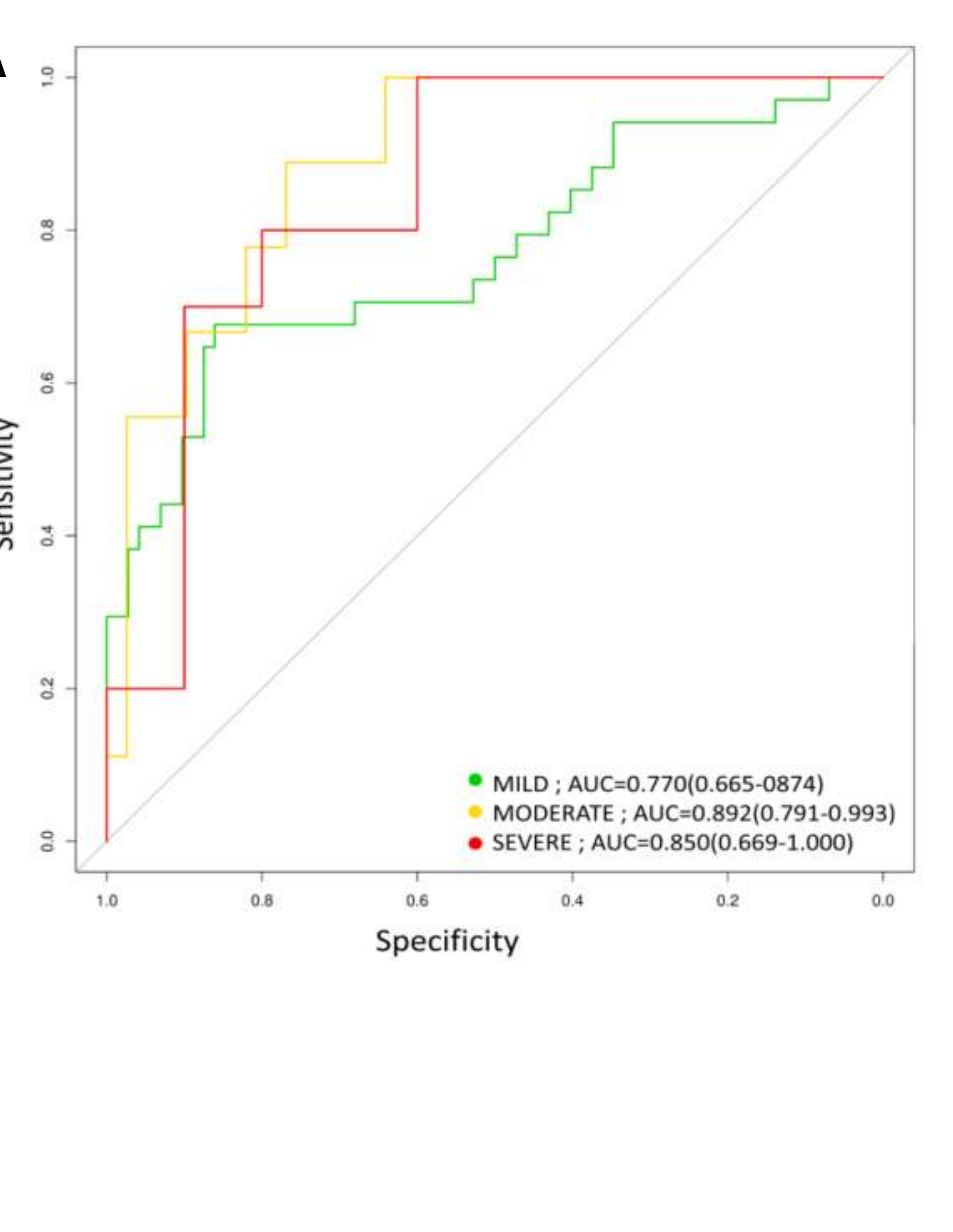

\section{Results}

Figure 2 Classification performance based on the 2-transcript DRS combined as [log2(FAM89expresion)ogerian of expression)]. (A) Box and whisker plot of DRS: the horizontal lines in the boxes indicate the thes the interquartilie range; the horizontal grey line is the DRS threshold that maximizes the AUC when comparing viral and bacterial patients. (B) ROC curves of different bacteria compared to rotavirus; the different A

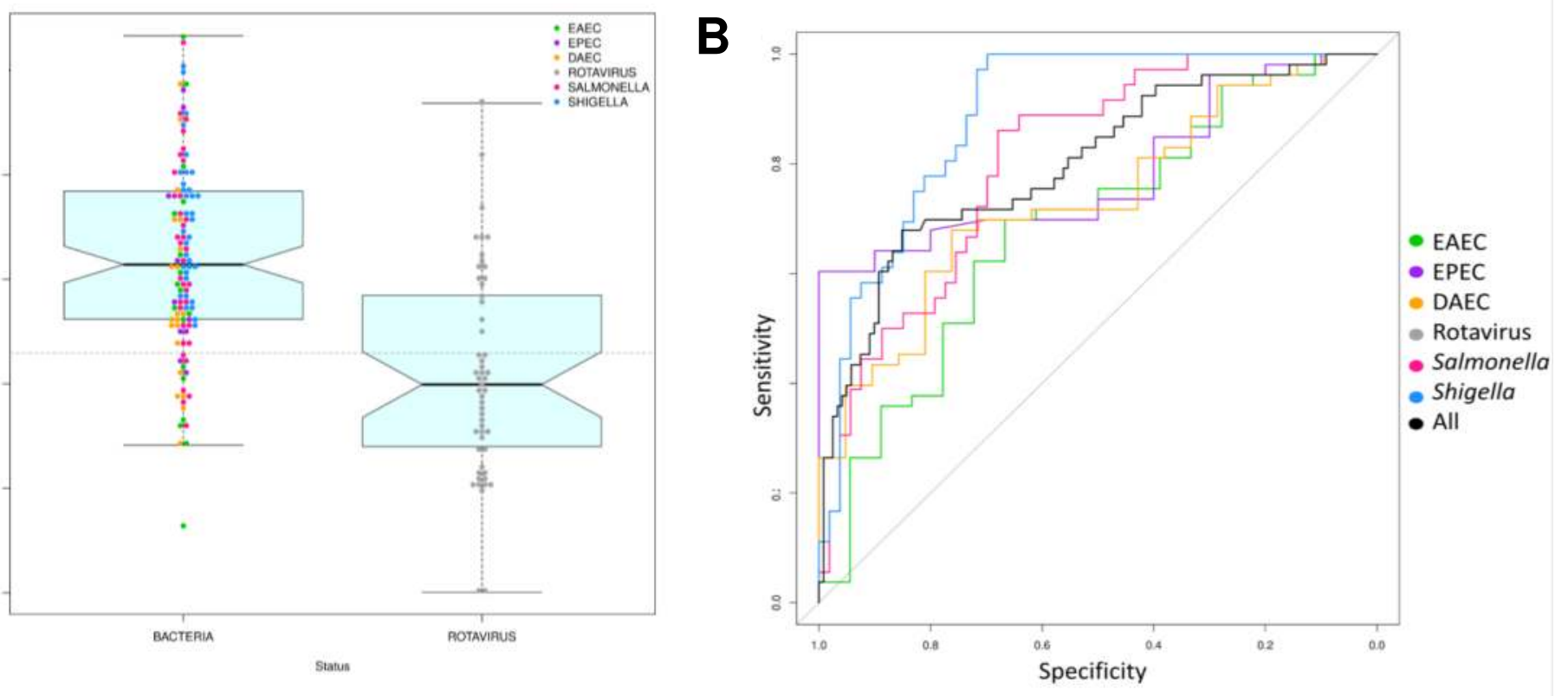

Figure 3 Classification performance based on the 2-transcript DRS combined as [log2(FAM89expresion)$\log 2($ LF44Lexpression)] and considering different levels of severity. (A) ROC curves of different bacteria
compared to rotavirus; AUC values by pathogen are provided in Table 1 ; and (B) and (C) box and whisker plots compared to rotavirus; AUC values by pathogen are provided in Taby

C

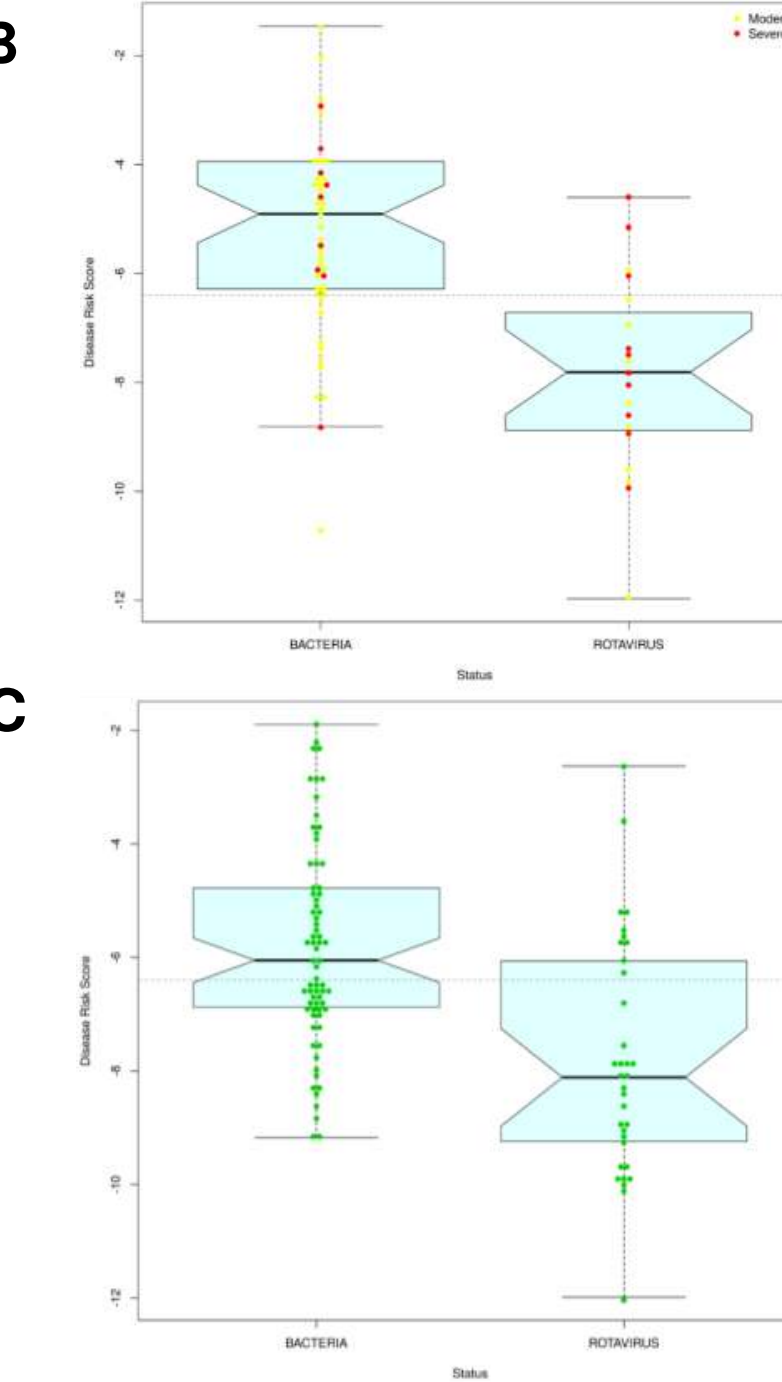

Table 1. AUC values and DRS for different pathogens. Figures in round brackets show the C. values surpessing a Bonferroni correction for multiple tests. Note that the reference vira

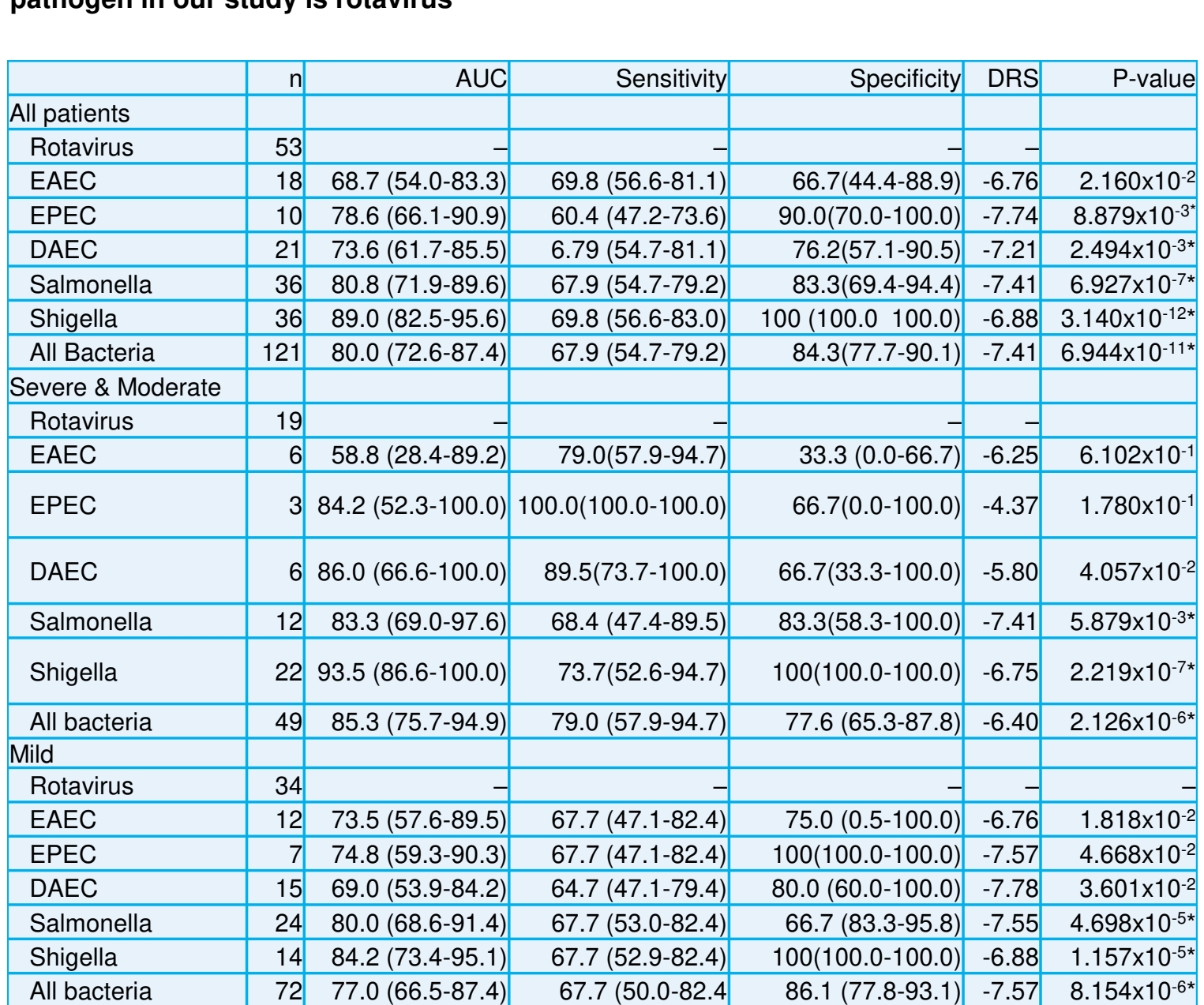

\section{Conclusions}

Just two transcripts seem to be enough to distinguish between

The 2-biomarker signature performs reasonably well in all of the evaluated settings, although its accuracy varies depending on the

More studies are needed before these transcriptomic biomakerks king. For this signature to be translated to

The first step is to evaluate the accuracy and scope of the test. As we did here

The second step would be to establish a mechanism that allow rapid and non expensive measure of the gene expression levels.

\section{References}

Herberg JA, Katorou M, Wright VJ, Shailes H, Elettherohorinou H, Hoggart CJ, Cebey-López M, Carter MJ,
Janes VA, Gormley S, Shimiziz C, Tremoulet AH, Barendregt AM, Salas A, Kanegaye J, Pollard AJ, Faust SN,

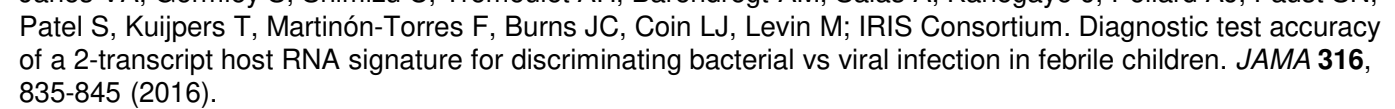

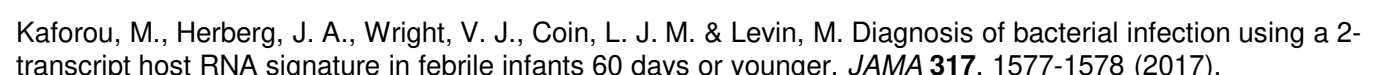
DeBerg, H. A. et al. Shared and organism-specilic host responses to childhood diarrheal diseases revealed by
whole blood transcript profiling, PLoS One 13 , 0192082 (2018).

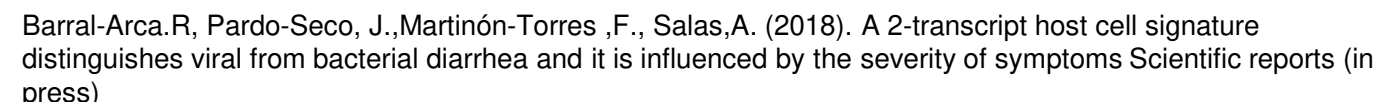

